Aim of the study: Large melanoma tumour caused arterial remodelling of the distal part of the great saphenous vein. The metastasis occurred at the site where inguinal lymphadenectomy was previously performed and the proximal part of the great saphenous vein was resected.

The aim of this study is the presentation of such a rare observation and literature overview concerning melanoma metastasis and possible stimuli causing remodelling of veins.

Material and methods: Macroscopic and microscopic analyses of the large blood vessel that supplies melanoma were made. The size and structure of the blood vessel was compared with the regular great saphenous vein. Results: The macroscopic examinations allowed us to ascertain that the blood vessel that was identified intraoperatively as the great saphenous vein, has a thick, stiff wall. The microscopic analysis allowed demonstrated that the tunica media was typical for a muscular artery morphology. The morphometric analysis revealed that the blood vessel wall in the area of metastatic tumour was much thicker than the wall of a regular great saphenous vein.

Conclusions: This malignant melanoma skin metastases caused the recanalisation of the great saphenous vein the lumen of which was obliterated during the initial surgical treatment The metastatic tumour supplied by large blood vessels grew extensively and caused arterial remodelling of the venous wall.

Key words: melanoma malignum melanoma metastases, great saphenous vein remodelling, muscular artery.

Contemp Oncol (Pozn) 2018; 22 (1): 54-59 DOI: https://doi.org/10.5114/wo.2018.74396

\section{Recanalization and remodeling of the great saphenous vein caused by the large melanoma's cutaneous metastasis}

\author{
Przemysław Stefaniak ${ }^{1}$, Przemysław Kwiatkowski², Zygmunt Kozielec ${ }^{3}$, \\ Janusz Godlewski ${ }^{1,2}$
}

'Department of Surgical Oncology Hospital Ministry of Internal Affairs with Warmia and Mazury Oncology Centre, Olsztyn, Poland

${ }^{2}$ Department of Human Histology and Embryology, School of Medicine, Collegium Medicum, University of Warmia and Mazury in Olsztyn, Poland

${ }^{3}$ Department of Pathomorphology, School of Medicine, Collegium Medicum, University of Warmia and Mazury, Olsztyn, Poland

\section{Introduction}

Melanoma is a malignant neoplasm that originates from melanocytes that are epidermis' chromocytes. The tumour starts to grow within the epithelial tissue, but it is not strictly classified to the carcinoma group because melanocytes in their embryonic development emanate from cells of the neural crests and have ectodermal origin [1]. The neoplastic phenotype features enable melanoma cells to migrate into the lumen of the blood and/or lymphatic vessels. The metalloproteinases, which are produced by melanoma cells, digest the vessels' walls and facilitate the cells' penetration into their lumen [2, 3]. Melanoma cells exhibit high metastatic potential and are transported by lymph or/and blood flow and form distant metastases along these vessels in target organs. There is strong diversity of the clinical course of melanoma, which strictly depends on the type of vessels through which the dissemination follows [4]. In the case of spread via lymphatic vessels, the formation of satellite tumours and the in transit metastases in the involved area of the skin are observed. Such cutaneous dissemination often occurs and is observed in almost $44 \%$ cases of malignant melanoma. Next the tumour occupies regional lymph nodes and subsequently spreads to distant organs: most often to the lungs, less often to the liver, brain, bone, and gastrointestinal tract [5]. In case of haematogenous spread, the melanoma's metastatic lesions are most commonly formed in the lungs, brain, and liver which directly affects the patient's poor prognosis [6].

\section{Aim of the study}

The purpose of this study is to present the cutaneous dissemination of a melanoma as a large tumour located on the thigh, which caused the recanalisation and arterial remodelling of the distal part of the great saphenous vein. The metastasis occurred in the site where inguinal lymphadenectomy was previously performed and the proximal part of the great saphenous vein was resected. The literature overview considers the analysis of vascularisation of melanoma metastasis and the evaluation of possible stimuli causing growth and remodelling of the vessels that supplied this tumour.

\section{Case report}

An 82-year-old patient (written consent for this analysis was obtained) was diagnosed with malignant melanoma, and the primary focus was localised on the heel of the right foot. Macroscopically the tumour surface was ulcerated, 

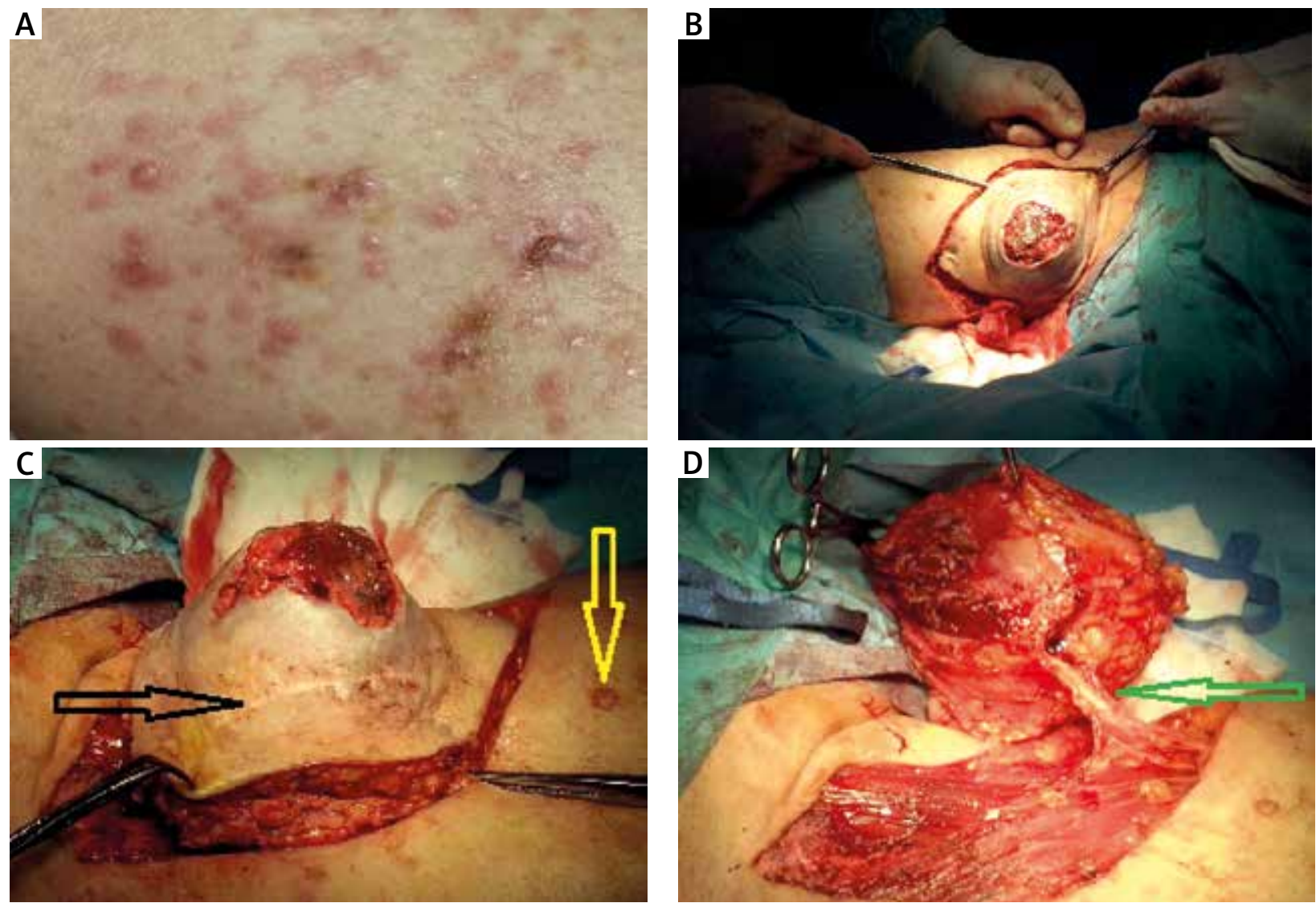

Fig. 1A-D. Photographs are made before and during the surgical treatment, the excision of large metastatic tumour located on the medial part of the right thigh. A) The micronodular cutaneous melanoma's dissemination on the patient's skin. B) The large tumour located on the medial side of the right thigh with the central, ulcerated part of the tumour. C) The scar (black arrow) after previous inguinal lymphadenectomy and the cutaneous melanoma metastasis (yellow arrow). The differences between the presented tumour and the numerous micronodular skin metastases are evident. D) The analyzed blood vessel (green arrow) extends from distal side of the tumour. Note, that the blood vessel has a typical anatomical localization for the great saphenous vein. The strong wall of this blood vessel is also noticeable. In the background, the compartment after the tumour excision with the anatomical structures is shown: the anterior and medial group of thigh muscles

and its diameter was $1.6 \mathrm{~cm}$. The tumour was surgically excised (RO); however, the procedure was not further radicalised. According to pathomorphological evaluation a nodular type of melanoma, Clark III and T4b stage was diagnosed. After 6 years, in 2014, relapse of the melanoma occurred in the area of the scar. Wide-margin resection of the tumour and sentinel node biopsy in the right inguinal region were performed. The histopathological analysis confirmed the relapse of melanoma in the region of the scar as well as the presence of lymph node metastasis. Subsequently the inguinal lymphadenectomy was performed, and, what should be emphasised, during that operation half of the upper femoral part of the great saphenous vein was resected. The histopathological examination revealed no neoplastic infiltration of the remaining inguinal lymph nodes. One year later, in 2015, the melanoma relapsed again in the region of the scar and another tumour resection was performed. After four months the micronodular melanoma's metastases occurred to the skin of the right lower limb (Fig. 1A). Moreover, pulmonary metastasis was revealed on CT scans. The patient was qualified for systemic chemotherapy. In June 2016, one of the skin's micronodular metastases started to enlarge significantly on the anterior-medial side of the right thigh and quickly grew to a large tumour. Chronic bleeding from the ulcerated surface of the tumour caused severe anaemia, and wide surgical resection of the tumour was performed. The patient recovered after surgery without any complications and was able to continue chemotherapy.

\section{Results}

During surgery a deep invasion of the soft tissues of the right thigh up to the adductor muscles compartment and the region of the right femoral artery and vein by the cancerous tumour was observed (Fig. 1B, C). During the dissection of tissues, a large blood vessel protruding from the distal end of the tumour was identified. The vessel was localised in a typical anatomical site for the great saphenous vein while macroscopically it presented the features of an artery, particularly its thick and stiff wall (Fig. 1D).

The pathomorphological (macroscopic) examinations allowed us to ascertain that the blood vessel that was identified intraoperatively as the great saphenous vein preserved a lumen that was gradually obliterated when the vessel penetrated into the tumour.

The standard haematoxylin and eosin (HE) histological sections of the blood vessel were made. The microscopic analysis with the use of an Olympus 41 microscope 

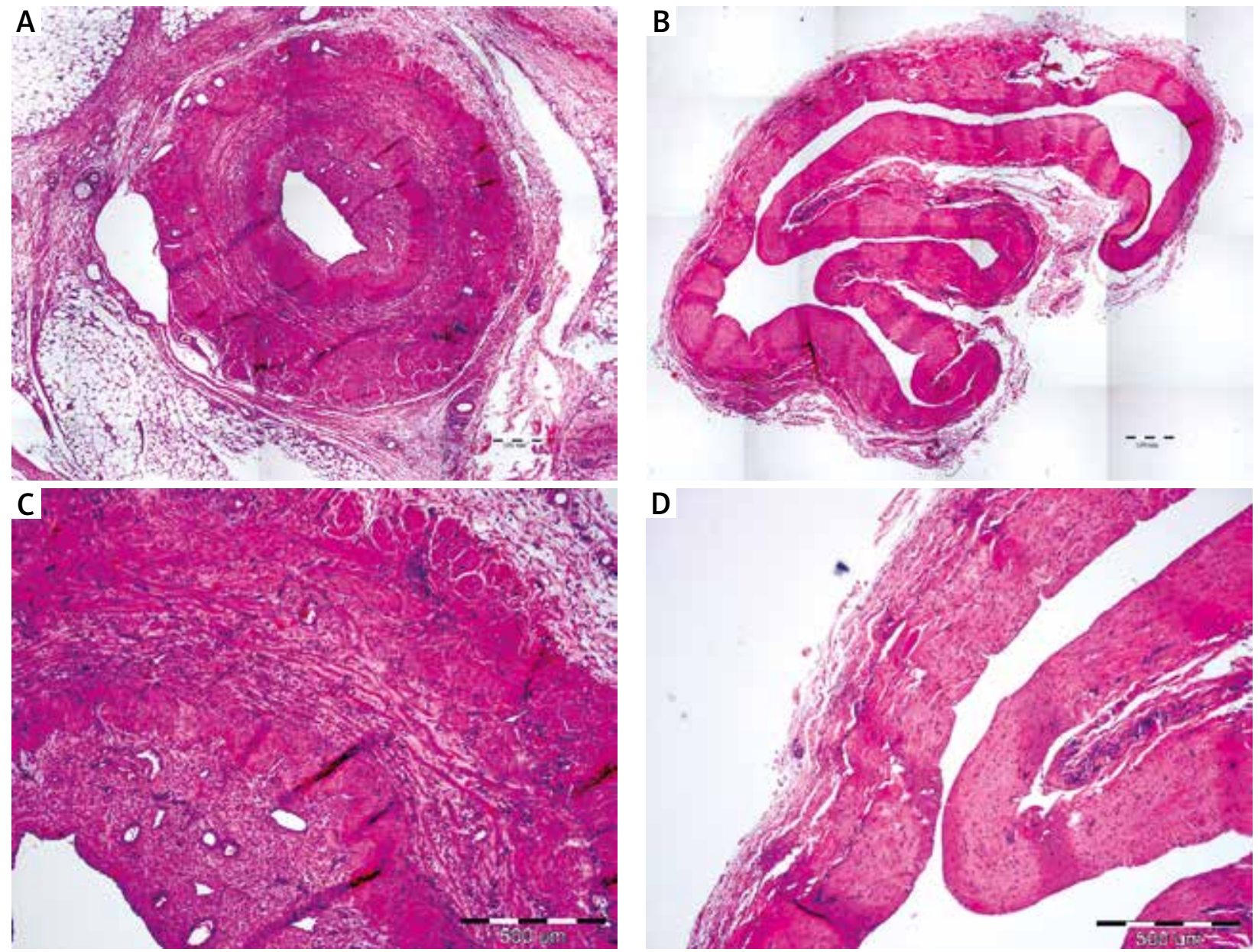

Fig. 2A-D. The picture presents the cross-section of the analyzed blood vessel, staining HE. The Fig. 2A and 2B are set up from several microscopic fields of view made at low magnification 40x. A, C) The blood vessel which supplies the analyzed tumour is well-seen, it is completely organized parietal thrombus of great saphenous vein which caused the thickening of the intima of the vessel. This vessel has features of a typical muscular artery: the shape of the lumen and the thickness of the wall, moreover numerous layers of smooth muscle cells in the tunica media and vasa vasorum in the tunica adventitia are visible. B, D) The regular great saphenous vein, note the differences in size and shape as well as the tunica media morphology between two compared blood vessels

equipped with an Olympus XC50 camera with appropriate computer software (Cell*) allowed us to observe a completely organised parietal thrombus of the great saphenous vein, which caused the thickening of the intima of the vessel. The lumen of the observed vein was lined with endothelium (simple squamous epithelium), it's tunica media was very thick, and the general morphology of the blood vessel was typical for a muscular artery (Fig. 2A). The structure of the analysed blood vessel was compared with the structure of a regular great saphenous vein, which was harvested during the surgical removal of varicose veins of a patient other than the one presented in this analysis (Fig. 2B).

The morphometric analysis revealed that the presented blood vessel's wall thickness in the area of metastatic tumour was $1780 \mu \mathrm{m}$ (Fig. 2D) and it was much thicker than the wall of a regular great saphenous vein, which was about $480 \mu \mathrm{m}$ thick (Fig. 2C). Microscopic observation demonstrated the presence of numerous vasa vasorum in the tunica adventitia of the blood vessel, which supplied the metastatic tumour. Moreover, immunohistochemical staining was made in order to identify the presence of muscle tissue (actin) and histochemical reaction to demonstrate the presence of elastic fibres (orcein) (Fig, 3A, C). Numerous layers of smooth muscle cells, a thick elastic lamina in the tunica media as well as a thin layer of connective tissue in the tunica adventitia were observed. These are the characteristic morphological features of a muscular artery. Immunohistochemical staining with the use of anti-CD31 antibody (Fig. 4A) and anti-CD34 antibody (Fig. 4B) were prepared, to demonstrate the presence of capillaries (vasa vasorum) within tunica media and tunica adventitia of the analysed blood vessel.

\section{Discussion}

This study presents a large metastasis to the skin, which caused the recanalisation of a part of the great saphenous vein, the lumen of which was previously obliterated during the inguinal lymphadenectomy. Metastases to the skin are referred to as satellite lesions (if localised a short distance from the primary tumour) or in-transit metastases (if the metastatic lesion appears between the primary tumour and the regional lymph nodes along the lymphatic vessels). 

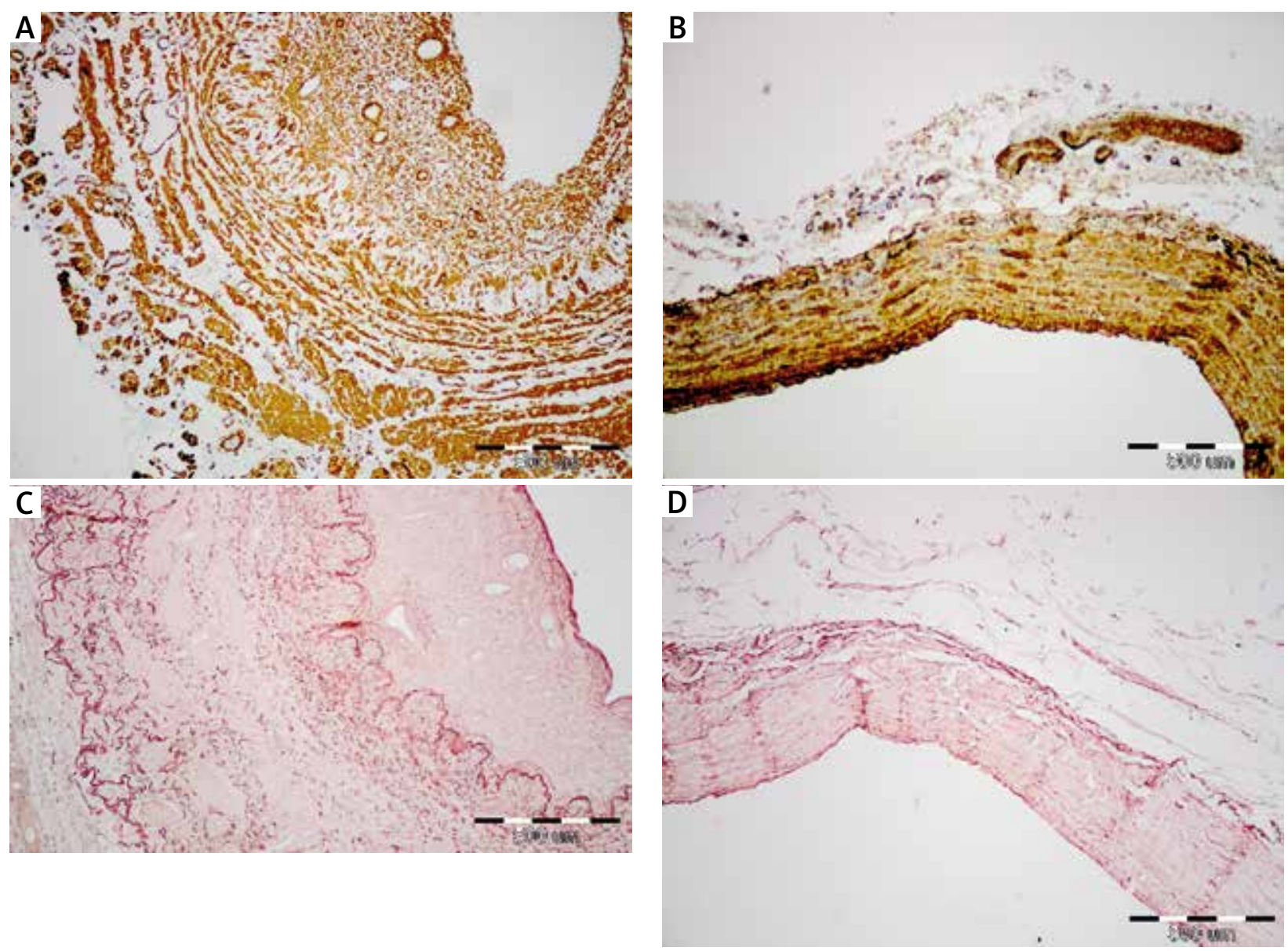

Fig. 3A-B. Immunohistochemical staining with the use of anti-actin antibody, to demonstrate smooth muscles in the vascular wall and a histochemical staining using orcein (B, C) for demonstration of elastic fibers presence. A, C) The cross - section of the blood vessel's wall which supplies the metastatic tumour. The picture presents the abundance of smooth muscle cells and elastic laminas in the tunica media, which is characteristic for a muscular artery morphology. B, D) The cross - section of the typical great saphenous vein shows a thin tunica media and a scarce presence of elastic fibers what are the typical features of vein's morphology

The phenomenon of in-transit metastases formation is a unique feature of malignant melanoma and at the same time is an unfavourable predictor of patient survival [7]. It is recognised that the formation of in-transit metastases may occur as a result of the arrest of tumour cells in the lumen of lymphatic vessels located within the dermis and subcutaneous tissue [8]. Other possible explanations for this phenomenon include the dispersion of tumour cells by tissue fluid as well as the spread of tumour cells along the outer surface of lymphatic and blood vessels, adjacent to pericytes [9]. The results of several recent studies have shown that the preferential formation of malignant melanoma metastases to the skin and subcutaneous tissue can be regulated by receptor-chemokine interactions, according to the "seed and soil" theory proposed by Stephen Paget. The binding of the CCR10 receptor, located on the surface of malignant melanoma cells, with its ligand located on the surface of normal epidermal keratinocytes, may be responsible for the formation of skin-specific metastases, and the selective blocking of this interference inhibits the formation of skin metastasis in the animal model [10].

In the presented patient, one of the in-transit metastases has grown extensively and the recurrent bleeding from its ulcerated surface resulted in significant anaemia. Bleeding and infection are the most common complications of malignant melanoma skin metastases and can significantly affect the patient's quality of life [11]. Bleeding from the ulcerating cancer tumours is associated with tumour angiogenesis, the process in which the newly formed blood vessels are characterised by substantially altered structure and function [12]. The process of angiogenesis is crucial for the progression and formation of solid tumours' metastases, including malignant melanoma. It has been shown that malignant melanoma cells produce large amounts of two major angiogenic stimulating factors: vascular endothelial growth factor (VEGF) and basic fibroblast growth factor (bFGF) [13]. The overexpression of VEGF in melanoma cells is induced by hypoxia and is dependent on $\alpha 1$ hypoxia inducible factor (HIF $\alpha 1$ ). The increase in bFGF concentration in the extracellular matrix is associated with digestion of extracellular matrix components by malignant melanoma metalloproteinases and the release of matrix-bound bFGF, which in turn stimulates endothelial cells' proliferation [14]. Another crucial pro-angiogenic growth factor in malignant melanoma is the placental growth factor (PIGF). PIGF is secreted both by pri- 

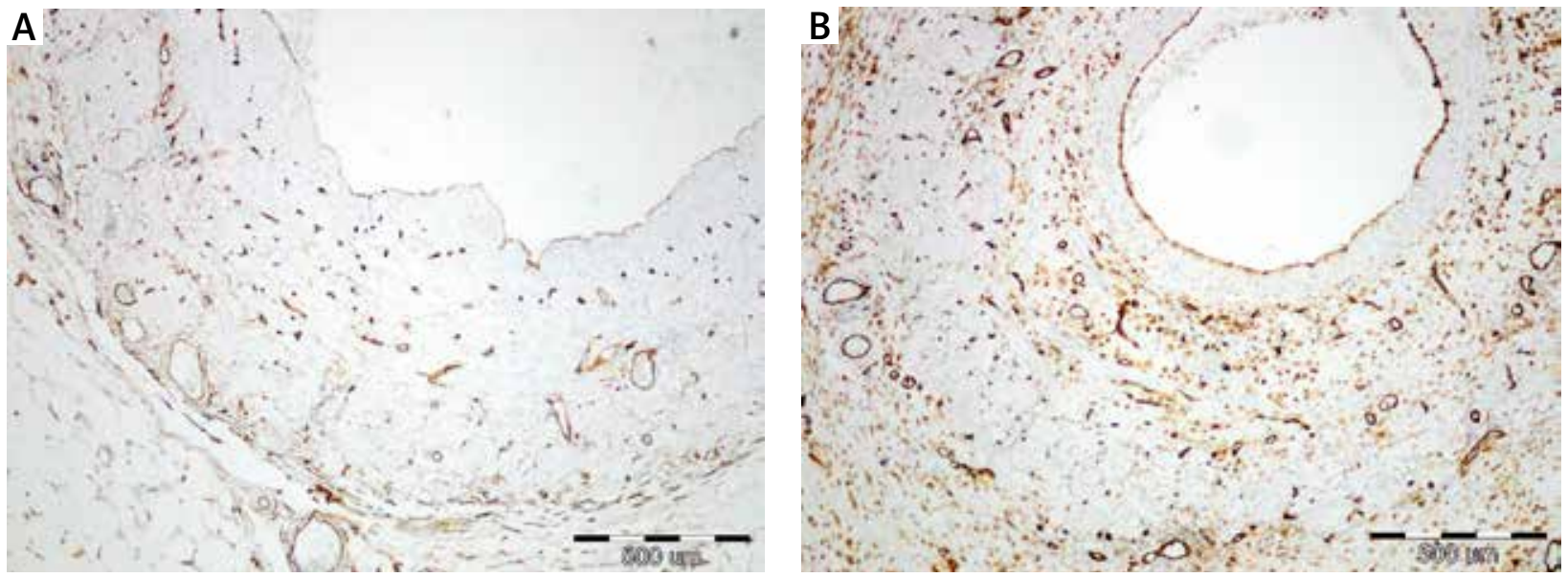

Fig. 4A-B. Immunohistochemical staining with the use of anti-CD31 antibody (A) and anti-CD34 antibody (B), to demonstrate endothelial cells in the analyzed blood vessel. The abundance presence of capillaries (vasa vasorum) within tunica media and tunica adventitia are clearly visible

mary and metastatic tumours and induces tumour cells' proliferation. It has been established that PIGF-induced neovascularization leads to rapid vascular branching and formation of blood vessels with larger diameter [15]. Other factors that may affect angiogenesis include interleukin 8 (IL-8), transforming growth factor (TGF) and urokinase plasminogen activator (UPA) [16]. Recently, a lot of attention has been devoted to vascular mimicry phenomenon, which due to the unsatisfactory results of antiangiogenic therapies implemented in the treatment of malignant melanoma, seems to be of particular importance. Malignant melanoma cells have a unique ability to acquire morphological and phenotypic characteristics of endothelial cells and to form channels connected to the host's blood circulation. These pseudovessels transport fluids that feed the tumour, leading to its uncontrolled growth. Vascular mimicry in malignant melanoma depends on the action of signaling molecules which are also involved in the process of embryonic vasculogenesis. It has been shown that the increased expression of ephrin type-A receptor 2 (EPHA2), laminin $5 \gamma 2$ and VE-cadherin in malignant melanoma cells is associated with these cells' ability of vascular mimicry [17]. In addition, the presence of PAS-positive loops and networks directly surrounding cancer cells, which are the hallmarks of malignant melanoma vascular mimicry, is associated with a worse prognosis in malignant melanoma patients [18]. Similarly, overexpression of EPHA2 and ephrin A1 correlates with vertical tumour growth, deeper tumour infiltration and shorter patients' survival [19]. A report on the microscopic analysis of the blood vessel, performed in the presented case, revealed its wall structure characteristic for an artery, and interestingly the blood vessel was anatomically located at the site where the great saphenous vein is usually found.

It has been shown that the thickness of the vascular wall within the metastatic tumour was significantly greater than that of the normal saphenous vein, and that the ultrastructure of the wall is typical of the muscular artery. The arterialisation of venous blood vessels is a well-known phenomenon during coronary artery by-pass grafting. The exposure of the vein graft to the arterial circulation leads to thickening of the vein's wall and widening of its diameter caused by the influx of blood under increased pressure [20]. Venous arterialisation is observed during formation of coronary artery bypasses, arterio-venous fistulas used to access the blood for haemodialysis treatment, or in the surgical treatment of erectile dysfunction. The molecular mechanisms leading to arterialisation of venous grafts exposed to higher blood pressure involve growth-factor-dependent cell proliferation, migration, and apoptosis as well as alterations in the production and degradation of extracellular matrix components. Activation of mitogen-activated protein kinases, 3-phosphatidylinositol kinase, and VEGF occurs [21-23].

\section{Conclusions}

This study presents the case of malignant melanoma skin metastases resulting in the recanalisation of the great saphenous vein, the lumen of which was obliterated during the initial surgical treatment. The metastatic tumour supplied by large blood vessels grew extensively and caused arterial remodelling of the venous wall. In the paper, the mechanisms of malignant melanoma metastasis formation and the phenomenon of venous arterialisation are discussed and corresponding microscopic images presented.

The authors declare no conflict of interest.

\section{Reference}

1. Ernfors P. Cellular origin and developmental mechanisms during the formation of skin melanocytes. Exp Cell Res 2010; 316: 1397 407.

2. Lugassy C, Zadran S, Bentolila LA, et al. Angiotropism, pericytic mimicry and extravascular migratory metastasis in melanoma: an alternative to intravascular cancer dissemination. Cancer Microenviron 2014; 7: 139-52.

3. Egeblad M, Werb Z. New functions for the matrix metalloproteinases in cancer progression. Nat Rev Cancer 2002; 2: 163-76. 
4. Meier F, Will S, Ellwanger U, Schlagenhauff B, Schittek B, Rassner G, Garbe C. Metastatic pathways and time courses in the orderly progression of cutaneous melanoma. Br J Dermatol 2002; 47: 62-70.

5. Leiter U, Meier F, Schittek B, Garbe C. The natural course of cutaneous melanoma. J Surg Oncol 2004; 8: 172-8.

6. Balch CM, Houghton AN, Sober AJ, Soong S. Cutaneous Melanoma. $4^{\text {th }}$ edition. Quality Medical Publishing, St. Louis 2003.

7. León P, Daly JM, Synnestvedt M, Schultz DJ, Elder DE, Clark WH Jr. The prognostic implications of microscopic satellites in patients with clinical stage I melanoma. Arch Surg 1991; 126: 1461-68.

8. Coit D, Ferrone C. Recurrent regional metastases of melanoma. In: Cutaneous melanoma. Balch C, Houghton AN, Sober AJ, Soong SJ, Atkins MA, Thompson JF (eds.). $5^{\text {th }}$ ed. Quality Medical Publishing, St Louis 2009; 487-98.

9. Wilmott J, Haydu L, Bagot M, et al. Angiotropism is an independent predictor of microscopic satellites in primary cutaneous melanoma. Histopathology 2012; 61: 889-98.

10. Damsky WE, Rosenbaum LE, Bosenberg M. Decoding melanoma metastasis. Cancers 2011; 3: 126-63.

11. Kaehler KC, Egberts F, Hauschild A. Electrochemotherapy in symptomatic melanoma skin metastases: intraindividual comparison with conventional surgery. Dermatol Surg 2010; 36: 1200-2

12. Jain RK. Normalization of tumour vasculature: an emerging concept in antiangiogenic therapy. Science 2005; 307: 58-62.

13. Vacca A, Ria R, Ribatti D, Bruno M, Dammacco F. Angiogenesis and tumour progression in melanoma. Recenti Prog Med 2000; 91: 581-7.

14. Ribatti D, Annese T, Longo V. Cancers (Basel). Angiogenesis and melanoma. 2010; 2: 114-32.

15. Luttun A, Autiero M, Tjwa M, Carmeliet P. Genetic dissection of tumour angiogenesis: are PIGF and VEGFR-1 novel anti-cancer targets? Biochim Biophys Acta 2004; 1654: 79-94.

16. Muehlenweg B, Sperl S, Magdolen V, Schmitt M, Harbeck N. Interference with the urokinase plasminogen activator system: a promising therapy concept for solid tumours. Expert Opin Biol Ther 2001; 1: 683-91.

17. Hendrix MJ, Seftor EA, Hess AR, Seftor RE. Vasculogenic mimicry and tumour-cell plasticity: lessons from melanoma. Nat Rev Cancer 2003; 3: 411-21.

18. Thies A, Mangold U, Moll I, Schumacher U. PAS positive loops and networks as a prognostic indicator in cutaneous malignant melanoma. J Pathol 2001; 195: 537-42

19. Easty DJ, Hill SP, Hsu MY, Fallowfield ME, Florenes VA, Herlyn M Bennett DC. Up-regulation of ephrin-A1 during melanoma progression. Int. J. Cancer 1999; 84: 494-501.

20. Henderson VJ, Cohen RG, Mitchell RS, et al. Biochemical (function al) adaptation of "arterialized" vein grafts. Ann Surg 1986; 203: 339-45.

21. Jalali S, Li YS, Sotoudeh M, Yuan S, Li S, Chien S, Shyy JY. Shear stress activates p60src-Ras-MAPK signaling pathways in vascular endothelial cells. Arterioscler Thromb Vasc Biol 1998; 18: 227-34.

22. Go YM, Boo YC, Park H, et al. Protein kinase B/Akt activates c-Jun $\mathrm{NH}(2)$-terminal kinase by increasing NO production in response to shear stress. J Appl Physiol 2001; 91: 1574-81.

23. Shay-Salit A, Shushy M, Wolfovitz E, Yahav H, Breviario F, Dejana E, Resnick N. VEGF receptor 2 and the adherents junction as a mechanical transducer in vascular endothelial cells. Proc Natl Acad Sci U S A 2002; 99: 9462-7.

\section{Address for correspondence}

\section{Janusz Godlewski}

Department of Human Histology and Embryology

School of Medicine, Collegium Medicum

University of Warmia and Mazury

Warszawska 30

10-900 Olsztyn, Poland

e-mail: janusz350@poczta.onet.pl

Submitted: 21.12.2017

Accepted: $\quad$ 17.01.2018 